\title{
Comparison of SPHC Hydrocode Results with Penetration Equations and Results of Other Codes
}

\author{
Steven W. Evans" and Roderick Stallworth ${ }^{\dagger}$ \\ NASA Marshall Space Flight Center, Huntsville, Alabama,35812 \\ Robert F. Stellingwerf $f^{*}$ \\ Stellingwerf Consulting, Los Alamos, New Mexico, 87544
}

The SPHC hydrodynamic code was used to simulate impacts of spherical aluminum projectiles on a single-wall aluminum plate and on a generic Whipple shield. Simulations were carried out in two and three dimensions. Projectile speeds ranged from $2 \mathrm{~km} / \mathrm{s}$ to 10 $\mathrm{km} / \mathrm{s}$ for the single-wall runs, and from $3 \mathrm{~km} / \mathrm{s}$ to $40 \mathrm{~km} / \mathrm{s}$ for the Whipple shield runs. Spallation limit results of the single-wall simulations are compared with predictions from five standard penetration equations, and are shown to fall comfortably within the envelope of these analytical relations. Ballistic limit results of the Whipple shield simulations are compared with results from the AUTODYN-2D and PAM-SHOCK-3D codes presented in a paper at the Hypervelocity Impact Symposium 2000 and the Christiansen formulation of 2003.

\section{Nomenclature}

$B H_{t} \quad=$ Brinnell hardness of the target material

$C \quad=$ speed of sound

$d_{c r i t} \quad=$ particle diameter marginally sufficient to produce failure

$E \quad=$ internal energy

$E_{h} \quad=$ Hugonio value of internal energy

$\gamma_{1} \quad=$ Grueneisen density factor

$\gamma_{G r m}=$ Grueneisen gamma factor

$K=$ numerical constant

$h \quad=$ inter-particle smoothing length

$m_{i} \quad=$ mass of particle $i$

$P_{h} \quad=$ Hugoniot value of pressure

$P_{i} \quad=$ pressure at particle $i$

$r_{i} \quad=$ position vector for particle $i$

$r \quad=\quad$ inter-particle separation scaled on smoothing length

$\rho \quad=$ material density

$S \quad=$ separation of plates of a Whipple shield

$S_{t} \quad=$ tensile strength of a shield plate

$t \quad=$ time, or, if a subscript, target

$t_{w} \quad=$ wall thickness in $\mathrm{cm}$

$v_{i} \quad=$ velocity of particle $i$

$v_{i j} \quad=$ relative velocity between particles $i$ and $j$

$V_{p} \quad=$ projectile velocity

$W_{i j} \quad=$ SPH particle mass smoothing kernel

\footnotetext{
- Aerospace Engineer, Environments Group, Mail Stop ED44, NASA, MSFC, Member AIAA.

+ Aerospace Engineer, Structures Group, Mail Stop ED23, NASA, MSFC, Member AIAA.

* Consultant, 2229 Loma Linda, Los Alamos, NM 87544, Member AIAA.
} 


\section{Introduction}

$\mathrm{T}$ HE use of hydrodynamic simulation codes to predict behavior of engineering structures under hypervelocity impact has become more common in recent years. These codes are especially valuable in extending predictions of system behavior beyond the velocity range commonly accessible to test facilities. In order to do so in a believable fashion, the predictions of a given code must first be compared with those of tests, accepted analytical prediction methods, and other hydrodynamic codes. In particular, the effect of the formulation and code implementation of the equation of state (eos) must be verified before predictions at extreme impact conditions are to be trusted. This paper describes a set of simulations undertaken to compare results using the SPHC hydrocode against well-established penetration equations for single-wall impacts, and, for Whipple shields, against a semianalytical expression and results from the AUTODYN-2D and PAM-SHOCK-3D codes.

\section{SPHC Code Description}

The code used for the present simulations is SPHC, a smooth particle hydrodynamics (SPH) code written in the C language. Each SPH particle consists of a fixed mass of material at a given location in space, together with a smoothing function, or kernel, that defines the particle's extent. The half-width of the kernel is the smoothing length, $h$, and is always taken to be the same in all defined directions. In these simulations a cubic B-Spline function similar to a Gaussian in shape was used as the smoothing kernel, $W_{i j}$ :

$$
\begin{aligned}
W i j & =K * 1.5\left(0.67-r^{2}+r^{3} / 2\right) & 0 & \leq r<1 \\
& =K * 0.25(2-r)^{3} & & 1 \leq r<2 \\
& =0 & & 2 \leq r
\end{aligned}
$$

Here $r=\left|\mathbf{r}_{\mathbf{i}}-\mathbf{r}_{\mathbf{j}}\right| / h$, and $K=\left(0.7 \pi h^{2}\right)^{-1}$

SPHC makes use of the Virtual Stress Point method of computing interparticle forces and states. In this method, as each particle is considered its nearest neighbors are identified and virtual stress points are created midway between the particle and each neighbor. Neighbor lists are normally rebuilt at each timestep using an octree technique. This method is faster than a simple $N^{2}$ particle search, scaling as $N \log N$. Typical initial values are about 16 neighbors per particle in 2-D simulations and 64 in 3-D. The particles carry the mass, temperature, and velocity information, while the interparticle deviatoric strains, stresses, strength model parameters, and fracture information are computed at the virtual stress points. By this method all stress forces are applied along the line of particle centers, rather than in directions determined by a stress tensor. Advantages of this approach are numerical stability, correct computation of rotations and translations, and applicability to thin shells and interfaces. It also allows a straightforward implementation of anisotropic strength models.

The material density is evaluated as the sum of masses weighted by the kernel functions:

$$
\rho_{i}=\sum_{j} m_{j} W_{i j}
$$

A "quiet start" correction begins the simulation with exactly zero pressure in all solid objects. Velocity updates are determined by conservation of momentum:

$$
\frac{\partial v_{i}}{\partial t}=-\sum_{j} m_{j}\left(\frac{P_{j}}{\rho_{j}^{2}}+\frac{P_{i}}{\rho_{i}^{2}}\right) d W_{i j}
$$

The pressure terms are computed from the equation of state, to which the artificial viscosity terms are added, and the material stress is subtracted.

Updates to the internal energy, E, are based on conservation of energy:

$$
\frac{\partial E_{i}}{\partial t}=\frac{P_{i}}{\rho_{i}^{2}} \sum_{j} m_{j} v_{i j} d W_{i j}
$$


Here $v_{i j}=\left(v_{i}-v_{j}\right)$ is the relative velocity. The $P_{i}$ contains only the thermal pressure, with the artificial viscosity and stress terms included in the summation, divided equally between particles $i$ and $j$. This is necessary to conserve energy exactly. The artificial viscosity used in SPHC follows the form developed by Monaghan ${ }^{1}$, having linear and quadratic terms.

The equation of state (EOS) used in these simulations was a Mie-Grueneisen analytic form, including phase changes from solid to liquid and vapor, plus an ionization model.

$$
P=P_{h}+\left[\gamma_{\text {Gran }}+\gamma_{1}\left(\frac{\rho_{0}}{\rho}\right)\right]^{*} \rho^{*}\left(E-E_{h}\right)
$$

The quantities $P_{h}$ and $E_{k}$ are the Hugoniot values, $\gamma_{G}$ is the Grueneisen coefficient, and $\gamma_{1}$ the Grueneisen density factor.

The EOS computation accepts the initial material density and temperature and computes the corresponding pressure, internal energy, phase, and sound speed. In subsequent steps, the pressures and temperatures are computed from the energy as given by the energy equation. The temperature is then used to determine the phase of the material, and the pressure computation changes as the material melts and vaporizes. Volumetric tension in the solid regime is limited by the minimum pressure parameter, usually taken to be the yield strength of the material.

At the melt temperature and above, the model approximates phase changes by a series of jumps in energy, with appropriate changes in the pressure law in each regime. Above the vaporization temperature the pressure is given by an ideal gas law. Six phase regimes are implemented:

$$
\begin{aligned}
& \text { solid } \quad \mathrm{T}=0 \rightarrow \mathrm{T}_{\text {met }} \\
& \text { liquid } \quad \mathrm{T}=\mathrm{T}_{\text {met }} \rightarrow \mathrm{T}_{\text {vap }} \\
& \text { mol vapor } \mathrm{T}=\mathrm{T}_{\mathrm{vep}} \rightarrow \mathrm{T}_{\mathrm{din}} \text { (if molecular) } \\
& \text { atomic vapor } \mathrm{T}=\mathrm{T}_{\mathrm{die}} \rightarrow \mathrm{T}_{\mathrm{low}} \\
& \text { ionizing } \mathrm{T}=\mathrm{T}_{\mathrm{bow}} \rightarrow \mathrm{T}_{\text {migh }} \\
& \text { fully ionized } \mathrm{T}>\mathrm{T}_{\text {tio }}
\end{aligned}
$$

The strength of materials model used in these simulations was a high-strain-rate-hardening model with fracture. This is a strain-to-fracture type of model with the fracture occurring at a specified strain and stress. The resulting stress/strain relation is then corrected to allow for the increased strength observed at high rates of strain. A more detailed discussion of the strength model is given in a companion paper in this session. Material properties needed in the simulations were obtained from the MatWeb.com website, http:/www.matweb.com/ during the period October 2003 to February 2004.

\section{Single-Wall Cases}

In the single-wall cases, the projectiles were 6061-T6 aluminum, and the plates were 2024-T81 with a thickness of $2 \mathrm{~mm}$. Projectiles impacted the plates at normal incidence. The failure criterion was the perforation or generation of detached spall from the back surface of the plate.

\section{A. Setup Details}

In all a total of thirty six individual scenarios were run. Nine were performed in three dimensions using version 7.05 of the code on a pc-type machine from October to December of 2003. The rest of the cases were two dimensional, using versions 7.05 and 7.10 of SPHC on three pc-type machines from January through March of 2004.

In making the V7.05 2-D runs, memory for up to 1,000 SPH particles was reserved, with the actual number of particles in a given case depending on the gridding and partitioning of the particles between the wall and projectile by the code. A minimum of 930 particles and a maximum of 968 particles were actually used. A "cylindrical" geometry was employed, so that the problem was simulated in the positive-X direction and mirrored across the $X=0$ axis. The 2-mm-thick target wall was 15 or $16 \mathrm{SPH}$ particles across, giving a wall particle size of $0.0133 \mathrm{~cm}$ or $0.0125 \mathrm{~cm}$. Penetrating projectiles were from $0.045 \mathrm{~cm}$ to $0.106 \mathrm{~cm}$, used from 14 to 60 particles each, and had 6 to 12 particles across the diameter. This gave an $S P H$ particle size range of $0.00750 \mathrm{~cm}$ to $0.01017 \mathrm{~cm}$. The ratio of projectile particle size to wall particle size ranged from 0.60 to 0.81 , so the two types of particles were of comparable sizes. 
A typical run is shown in Figure 1. This is the "borderline" no-penetration case for $7 \mathrm{~km} / \mathrm{s}$. Colors are green=solid, light green = plastic deformation, blue = fractured, and red = melted. Note that a spall "cone" has begun to form in this case, but does not penetrate to the rear surface.

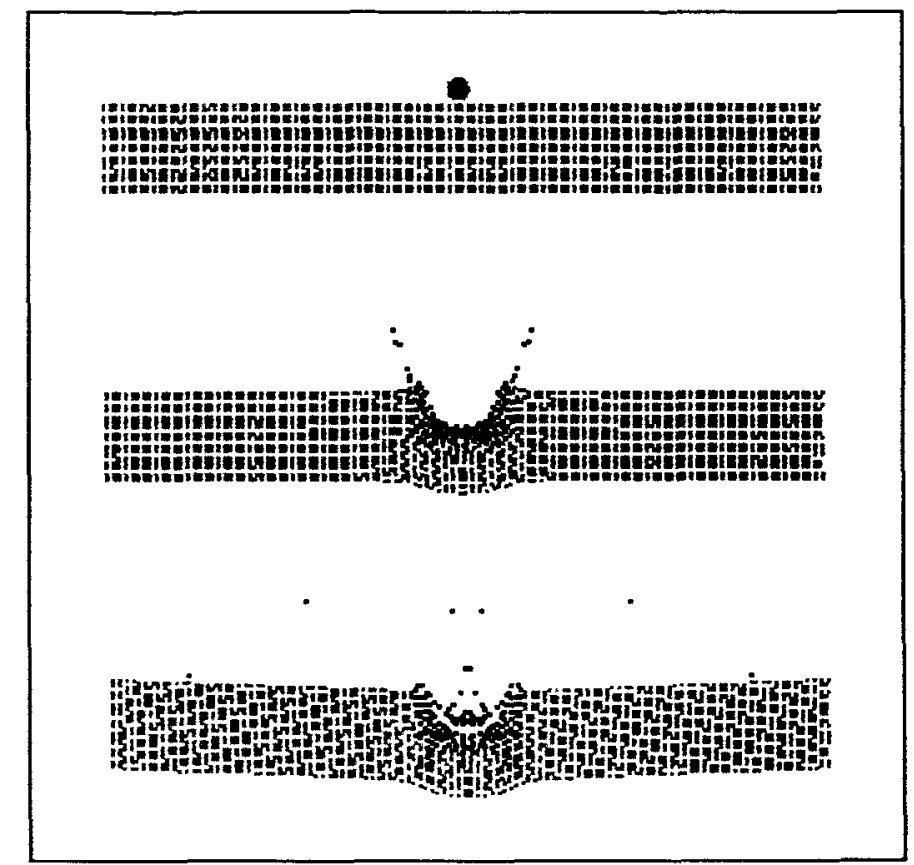

Figure 1: The case $V=7 \mathrm{~km} / \mathrm{s}, \mathrm{d}=0.5 \mathrm{~mm}$ shown at $0,1,10 \mu \mathrm{s}$.

For the V7.10 2-D runs memory was reserved for up to 5,000 particles. The scenario geometry was cylindrical as before. The actual particle counts ranged between 4,496 and 4,634. The wall was 34 or 35 particles across, giving a wall particle size of $0.00571 \mathrm{~cm}$ or $0.00588 \mathrm{~cm}$. Penetrating projectiles were from $0.046 \mathrm{~cm}$ to $0.097 \mathrm{~cm}$ in diameter, used from 60 to 234 particles each, and had 12 to 24 particles across the diameter. This gave an SPH particle size range of $0.00364 \mathrm{~cm}$ to $0.00419 \mathrm{~cm}$. The ratio of projectile particle size to wall particle size ranged from 0.64 to 0.73 , so, again, the two types of particles were of comparable sizes.

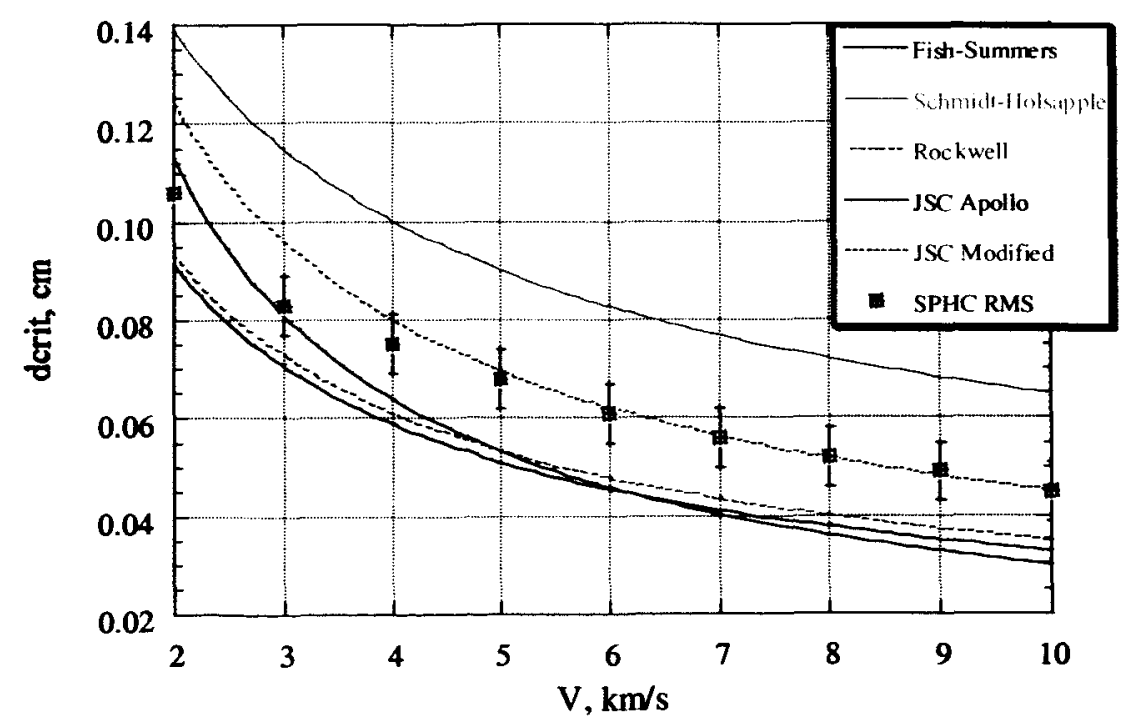

Figure 2: Single-wall spallation limit comparison - SPHC vs. standard penetration equations. 
The emphasis on similar particle size is due to the dependence of simulation results on the compatibility of the interacting particles. If one set is much smaller than another, some of the smaller ones may "slip through the grid" of the larger type, producing erroneous results. Another consideration is the effect of using a cylindrical geometry, which is in some sense singular about the symmetry axis: particles moving toward this axis may find themselves constrained - by their neighbors and the impenetrability of the axis - to move along that axis. Although axiallytraveling projectile fragments are observed in tests, the degree to which such fragments are realistically simulated in a cylindrically symmetric scenario is open to question. For the current set of computations, the results were carefully examined for axis problems, and we believe that no serious problems are present.

\section{B. Single-Wall Results}

Minimum projectile diameters necessary to produce failure $\left(d_{\text {erit }}\right)$ over the speed range of $2 \mathrm{~km} / \mathrm{s}$ to $10 \mathrm{~km} / \mathrm{s}$ were determined as root-mean-square values from the results of the thirty six cases. The resulting spallation limit data are shown in Fig. 2 and Table 1, where they are compared with predictions from five accepted penetration relations ${ }^{2}$ : the Fish-Summers, Schmidt-Holsapple, Rockwell, JSC Apollo, and JSC Modified Cour-Palais penetration equations. The error bars on the SPHC data in the figure are $\pm 0.006 \mathrm{~cm}$, indicating the largest root-mean-square variation encountered among these cases.

The SPHC results fall comfortably within the envelope given by the five standard relations, agreeing most closely with the JSC Modified expression for the spallation limit:

$$
d_{\text {cris }}^{J S C M}=\left[0.01735\left(B H_{t}\right)^{1 / 4}\left(\rho_{t} / \rho_{p}\right)^{1 / 2}\left(C_{t} / V_{p}\right)^{2 / 3} t_{w}\right]^{18 / 19}
$$

Substituting the material property values for the alloy 6061-T6 projectile and 2024-T81 plate gives:

$$
d_{\text {crit }}^{J S C M}=0.19234\left(V_{p}\right)^{-0.6316}
$$

The SPHC results can be fitted by the power law expression:

$$
d_{c r i t}^{\text {SPC }}=0.15106\left(V_{p}\right)^{-0.51334}
$$

for which the correlation coefficient is 0.99748 . As seen in Fig. 1, SPHC is most conservative in the velocity range of $2 \mathrm{~km} / \mathrm{s}$ to $3 \mathrm{~km} / \mathrm{s}$ where its predictions are close to those of the Fish-Summers relation. This may be due to the "axial fragment" phenomenon. Above $4 \mathrm{~km} / \mathrm{s}$ its predictions can be termed "moderate."

\begin{tabular}{|c|c|c|c|c|c|c|c|}
\hline $\mathrm{V}, \mathrm{km} / \mathrm{s}$ & F-S & S-H & Rock & JSC A & JSC M & SPHC & Fitted \\
\hline 2.0 & 0.113 & 0.139 & 0.093 & 0.091 & 0.124 & 0.106 & 0.106 \\
\hline 3.0 & 0.081 & 0.115 & 0.073 & 0.071 & 0.0 & 0.083 & 0.086 \\
\hline 4.0 & 0.064 & 0.100 & 0.061 & 0.059 & 0.080 & 0.075 & 0.074 \\
\hline 5.0 & 0.053 & 0.090 & 0.053 & 0.051 & 0.070 & 0.068 & 0.066 \\
\hline 6.0 & 0.046 & 0.083 & 0.048 & 0.045 & 0.062 & 0.061 & 0.060 \\
\hline 7.0 & 0.040 & 0.077 & & 0.0 & 0.056 & 0.056 & 0.056 \\
\hline 8.0 & 0.036 & 0.072 & 0.040 & 0.038 & 0.052 & 0.052 & 0.052 \\
\hline 9.0 & 0.033 & 0.068 & 0.037 & 0.035 & 0.048 & 0.049 & 0.049 \\
\hline 10. & 0.030 & 0.065 & 0.035 & 0.033 & 0.045 & 0.045 & 0.046 \\
\hline
\end{tabular}

Table 1: Single-wall Spallation Limits

$$
\mathrm{d}_{\mathrm{cril}}, \mathrm{cm}
$$




\section{Whipple Shield Cases}

In the Whipple shield cases, the shield configuration of Palmieri et al. ${ }^{3}$ was adopted, consisting of a bumper of 2024-T81 aluminum of thickness $1.6 \mathrm{~mm}$, a standoff of $120 \mathrm{~mm}$, and a backwall of 2219-T87 of thickness $3.2 \mathrm{~mm}$. Projectiles were spheres of 2024 which impacted the bumper at normal incidence. The failure criterion was perforation of or detached spall from the back of the back wall. Comparisons are made with the AUTODYN-2D and PAM-SHOCK-3D results of Palmieri et al., and with the predictions of the generic Whipple shield penetration equation of Christiansen ${ }^{4}$. These comparisons are shown in Fig. 4 and Table 2. The SPHC results are to be considered as preliminary in nature, since a more detailed investigation of the materials' behavior and the effects of scenario geometry is planned.

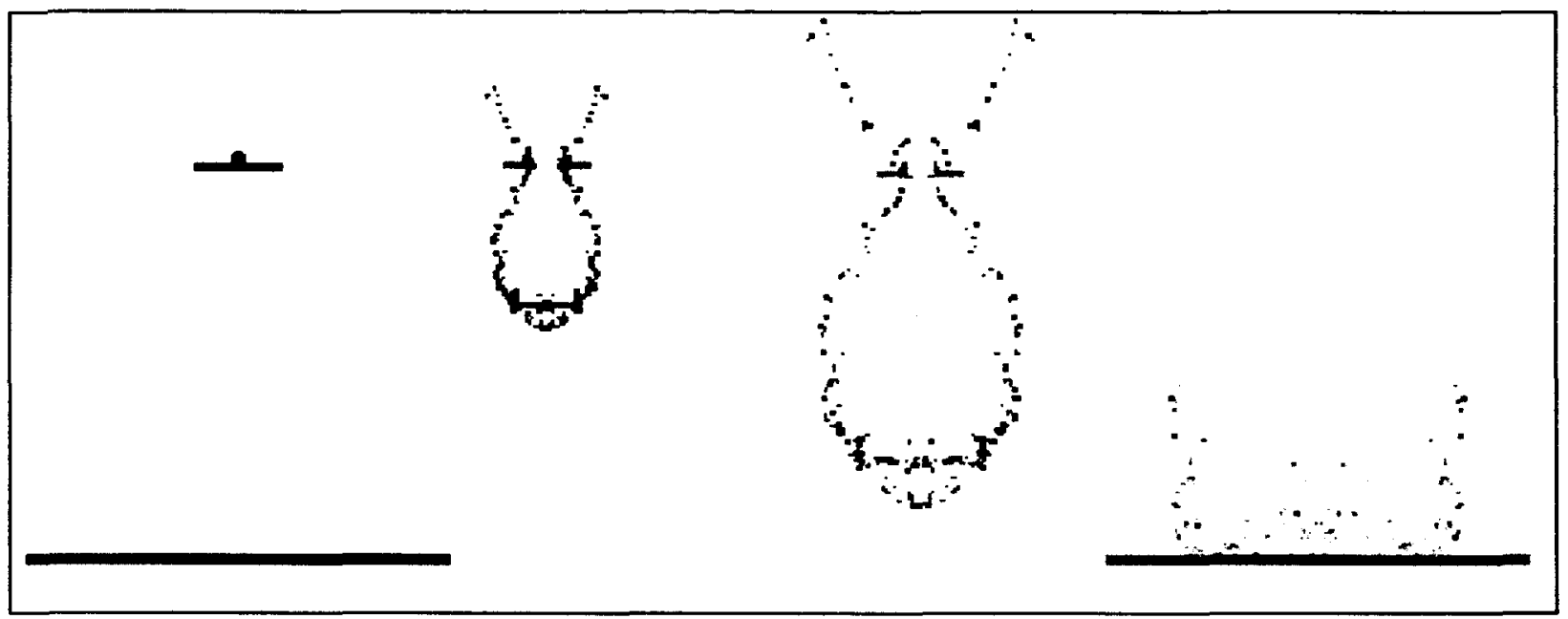

Figure 3: The case $V=7 \mathrm{~km} / \mathrm{s}, \mathrm{d}=3 \mathrm{~mm}$ at $0,10,20,30 \mu \mathrm{s}$.

\section{A. Setup Details}

A total of thirteen 2-D, cylindrical symmetry scenarios were examined using version 7.07 on three pc-type machines during January and February of 2004. Memory for up to 6,000 SPH particles was reserved. A minimum of 5,229 particles and a maximum of 5,531 particles were actually used. The 1.6-mm-thick bumper was 9 SPH particles across, giving a bumper particle size of $0.0178 \mathrm{~cm}$. The $3.2-\mathrm{mm}$-thick backwall was $15 \mathrm{SPH}$ particles across, giving a wall particle size of $0.0213 \mathrm{~cm}$. The ratio of bumper particle size to backwall particle size was 0.84 . Penetrating projectiles were from $0.30 \mathrm{~cm}$ to $0.66 \mathrm{~cm}$ in diameter, used from 132 to 572 particles each, and had 18 to 38 particles across the diameter, which gave an $S P H$ particle size range of $0.0160 \mathrm{~cm}$ to $0.0178 \mathrm{~cm}$. The ratio of projectile particle size to bumper particle size ranged from 0.90 to 1.00 ; the projectile particle size to wall particle size ratio ranged from 0.75 to 0.84 . Again, the three types of particles were of comparable sizes.

Figure 3 shows a sequence of snapshots from the non-penetrating case velocity=7 km/s, diameter $=3 \mathrm{~mm}$. for the Whipple Shield case. Except for the first image, which is entirely solid, the colors are green = solid, blue = fractured solid, and red = liquid. In the last frame, the yellow/green/red particles are a mixture of liquid and vapor particles At higher velocities many of the models exhibited a phase change to vapor upon collision with the lower shield.

The computation can be set to put the lower layer "on hold" until late in the run, and drop the upper layers when they no longer interact with the downward moving material. Both of these features can be seen in the figure. In addition, in some runs "absorb" boundaries are used at the outer edges of the lower target to discard particles that have left the vicinity of the interaction regions. These techniques are used solely to save computational time and do not affect the final result in any way. 


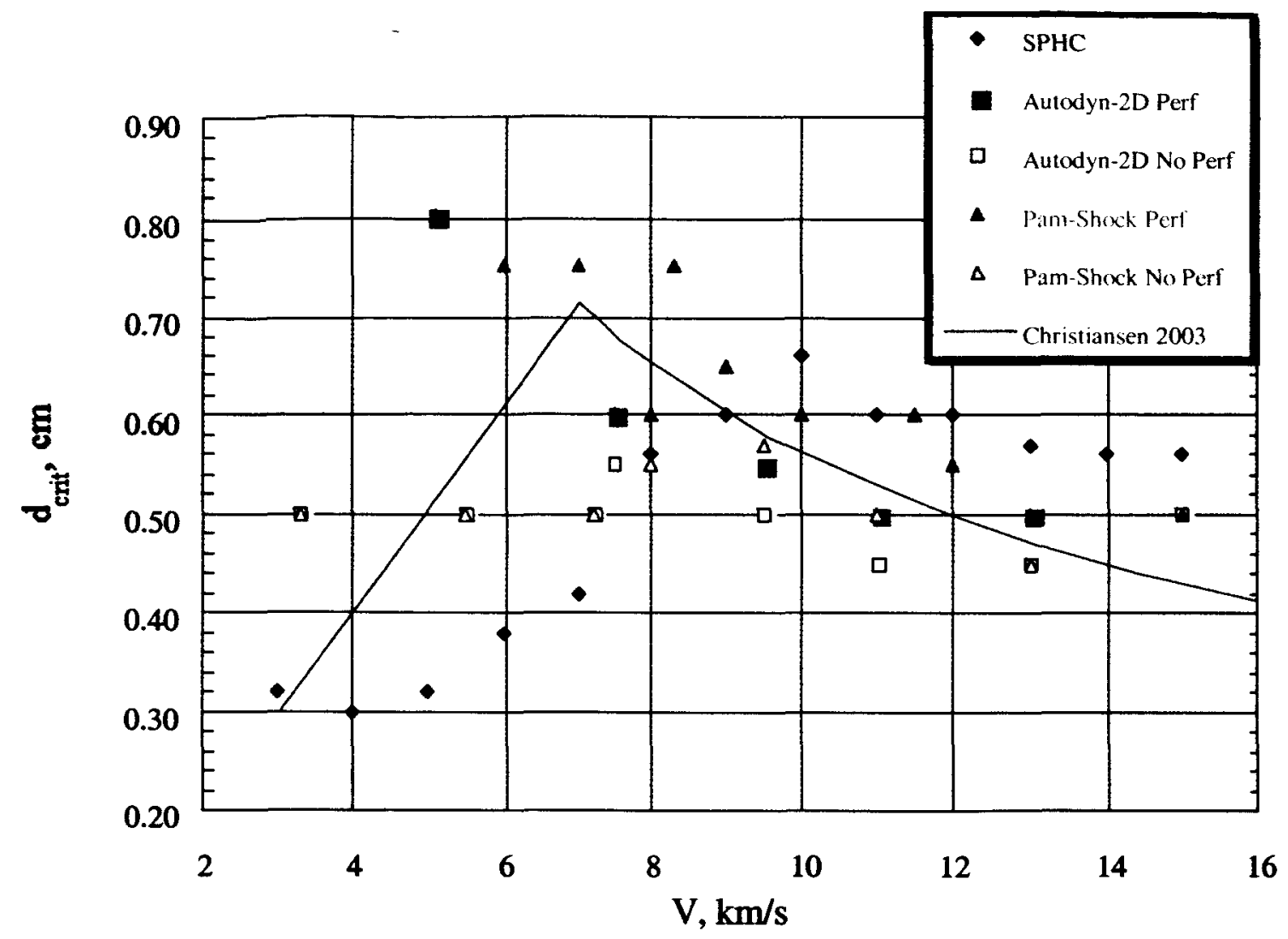

Figure 4: Whipple shield ballistic limit data from SPHC, AUTODYN, PAM-SHOCK, and Christiansen.

\section{B. Whipple Shield Results: Medium Velocities $-3 \mathrm{~km} / \mathrm{s}$ to $7 \mathrm{~km} / \mathrm{s}$}

The SPHC results are considerably more conservative at velocities between $3 \mathrm{~km} / \mathrm{s}$ and $7 \mathrm{~km} / \mathrm{s}$ than Christiansen's expression in this range. They show a minimum in the critical projectile size of $0.30 \mathrm{~cm}$ at a velocity of $4 \mathrm{~km} / \mathrm{s}$, whereas Christiansen's relation trends linearly upward from $3 \mathrm{~km} / \mathrm{s}$, and predicts a critical diameter at 4 $\mathrm{km} / \mathrm{s}$ of $0.40 \mathrm{~cm}$. SPHC's $d_{\text {crit }}$ 's continue to fall well below the Christiansen line for the remainder of the medium velocity range, being only 59 percent of Christiansen's value at $7 \mathrm{~km} / \mathrm{s}$.

The PAM-SHOCK data below $7 \mathrm{~km} / \mathrm{s}$ only roughly bracket the critical size range. This may be due to the roughness of the "search grid" Palmieri et al. employed in using this code due to large run times required, lack of an axial symmetry option, difficulties they encountered in determining perforations reliably, and the fact that they were interested in examining the high velocity regime. In no case did they use PAM-SHOCK to examine projectiles closer together in size than $0.05 \mathrm{~cm}$. PAM-SHOCK predicts no perforation by a $0.50 \mathrm{~cm}$ sphere at $3.3 \mathrm{~km} / \mathrm{s}$, a result contradicted by both the SPHC data and Christiansen's expression.

Neither AUTODYN nor PAM-SHOCK predicts perforation at $3 \mathrm{~km} / \mathrm{s}$ for projectiles of $0.5 \mathrm{~cm}$ size, whereas SPHC's marginal perforation diameter is $0.32 \mathrm{~cm}$ at this speed; Christiansen's intermediate velocity expression predicts perforation by a $0.30 \mathrm{~cm}$ sphere.

The crucial quantity in this velocity range is the degree of fracture of the original projectile after it encounters the upper shield. Experiments usually show a central fragment or fragments that will pose the main threat to the lower wall. The SPHC models show this behavior, but do have problems resolving the on-axis fragments due to resolution and numerical problems near the axis. This may lead to excess penetration. Future work with finer resolution and 3D models should resolve this issue. In any case, we estimate the error bars for the results in this range to be about +1 $0.05 \mathrm{~cm}$, or an uncertainty of about $1 \mathrm{~mm}$ in the penetration diameter. 


\section{Whipple Shield Results: High Velocities $-7 \mathrm{~km} / \mathrm{s}$ to $20 \mathrm{~km} / \mathrm{s}$}

Above $7 \mathrm{~km} / \mathrm{s}$ the SPHC results first approach the Christiansen curve, cross it at about $9 \mathrm{~km} / \mathrm{s}$, and then remain above the earlier estimates, consistently, at higher velocities. Figure 5 shows the case velocity $=14 \mathrm{~km} / \mathrm{s}$, diameter $=$ $5 \mathrm{~mm}$ at $13 \mu \mathrm{s}$. This is a case that should fail, according to the earlier results. Here green $=$ solid, blue $=$ fractured solid, yellow = liquid, red = vapor. The arrows indicate the direction of flow of the material at this time. The majority of the initially impacting material has "flashed" into a vapor or near-vapor state. This hot vapor does not damage the lower shield, and, in fact can interact with incoming fragments, reducing their effectiveness at penetrating the shield. In some tests the incoming high density material can experience a fluid instability when decelerated by the lower density hot cloud formed from previously arriving debris, further degrading its effectiveness to penetrate the lower layer. The outcome is that the lower shield survives.

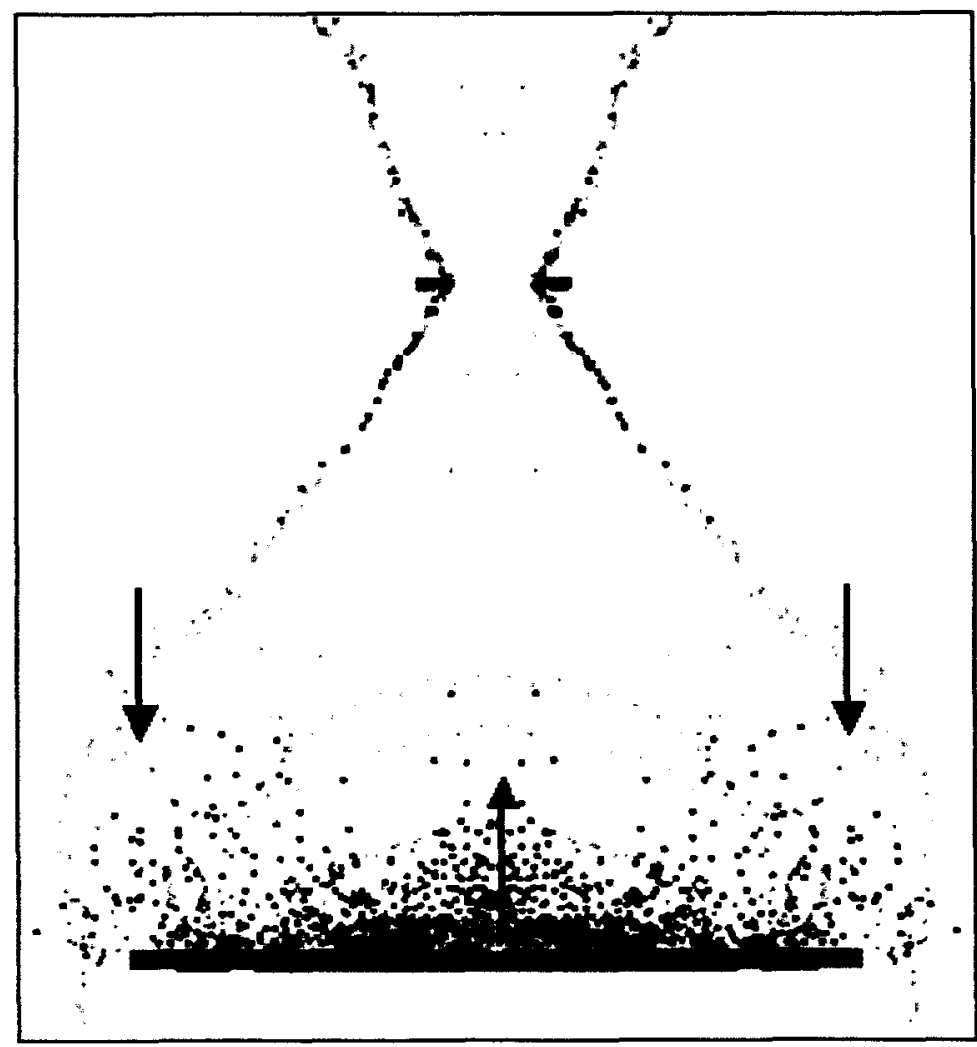

Figure 5: Case $V=14 \mathrm{~km} / \mathrm{s}, \mathrm{d}=5 \mathrm{~mm}$, at $13 \mu \mathrm{s}$.

\section{Whipple Shield Results: Very High Velocities - above $20 \mathrm{~km} / \mathrm{s}$.}

For very high velocities, a simple argument holds that the details of the shield should not matter, and the $-2 / 3$ scaling for high energy impacts should again appear. The Christiansen curve assumes this transition at $7 \mathrm{~km} / \mathrm{s}$. The SPHC results show some indication of approaching this limit at about $10 \mathrm{~km} / \mathrm{s}$, but the vapor mechanism described above could in principle shift the curve to even higher velocities. In addition, at about $25-30 \mathrm{~km} / \mathrm{s}$ the upper shield has the capability of vaporizing the impacting projectile. SPHC has physics capable of handling high temperature gas, including ionization. A few tests were run with $5 \mathrm{~mm}$ diameter projectiles at velocities of $20-40 \mathrm{~km} / \mathrm{s}$. None penetrated. This means that the ballistic curve is flat out to $40 \mathrm{~km} / \mathrm{s}$.

Since the diameter of the debris cloud when it strikes the lower plate is always equal to about the distance between the plates, we can easily estimate the velocity at which the momentum of a $5 \mathrm{~mm}$ aluminum particle will deliver an impulse equal to the tensile strength of the aluminum lower plate. This answer turns out to be about $\mathbf{5 0}$ $\mathrm{km} / \mathrm{s}$ [transit time $\mathrm{t}=\mathrm{S} / \mathrm{v}$, implies $\mathrm{S}_{\mathrm{r}} \mathrm{S}^{3}=\mathrm{mv^{2 }}$ ]. At this velocity simple momentum transfer should be able to damage the lower plate and the curve will begin to drop. With this scaling, $d_{\text {crit }}$ depends once again on velocity to the $-2 / 3$ power, but it varies linearly with the separation of the plates, rather than the square root dependence found at lower velocities. 
Table 2: Whipple Shield Ballistic Limits

\begin{tabular}{|c|c|c|c|c|c|c|}
\hline $\mathrm{V}, \mathrm{km} / \mathrm{s}$ & & & & rit, cm & & \\
\hline & SPHC & Auto perf & Auto no & Pam perf & Pam no & Christiansen \\
\hline 3.0 & 0.32 & & & & & 0.300 \\
\hline 3.3 & & & 0.50 & & 0.50 & \\
\hline 3.5 & & & & & & 0.352 \\
\hline 4.0 & 0.30 & & & & & 0.403 \\
\hline 4.5 & & & & & & 0.455 \\
\hline 5.0 & 0.32 & & & & & 0.506 \\
\hline 5.1 & & 0.80 & & 0.80 & & \\
\hline 5.5 & & & 0.50 & & 0.50 & 0.558 \\
\hline 6.0 & 0.38 & & & 0.75 & & 0.609 \\
\hline 6.5 & & & & & & 0.661 \\
\hline 7.0 & 0.42 & & & 0.75 & & 0.712 \\
\hline 7.2 & & & 0.50 & & 0.50 & \\
\hline 7.5 & & 0.60 & 0.55 & 0.60 & & 0.680 \\
\hline 8.0 & 0.56 & & & 0.60 & 0.55 & 0.652 \\
\hline 8.3 & & & & 0.75 & & \\
\hline 8.5 & & & & & & 0.626 \\
\hline 9.0 & 0.60 & & & 0.65 & & 0.602 \\
\hline 9.5 & & 0.56 & 0.50 & & 0.55 & 0.581 \\
\hline 10.0 & 0.66 & & & 0.60 & & 0.561 \\
\hline 10.5 & & & & & & 0.543 \\
\hline 11.0 & 0.60 & 0.50 & 0.45 & & 0.50 & 0.527 \\
\hline 11.5 & & & & 0.60 & & 0.511 \\
\hline 12.0 & 0.60 & & & 0.55 & & 0.497 \\
\hline 12.5 & & & & & & 0.484 \\
\hline 13.0 & 0.57 & 0.50 & 0.45 & 0.50 & 0.45 & 0.471 \\
\hline 13.5 & & & & & & 0.460 \\
\hline 14.0 & 0.56 & & & & & 0.449 \\
\hline 14.5 & & & & & & 0.438 \\
\hline 15.0 & 0.56 & & 0.50 & 0.50 & & 0.428 \\
\hline 15.5 & & & & & & 0.419 \\
\hline 16.0 & & & & & & 0.410 \\
\hline
\end{tabular}

\section{Conclusion}

A preliminary set of SPHC models has been run for both single-walled and double walled meteoroid shield configurations. The single wall results are in good agreement with previously obtained relations. The double walled (Whipple) models show trends similar to earlier results, but deviate in several significant respects. First, the SPHC results in the intermediate velocity range $(3-7 \mathrm{~km} / \mathrm{s})$ fall below the usual curve, indicating that smaller projectiles were penetrating than expected. Second, the SPHC results in the high velocity range $(V>7 \mathrm{~km} / \mathrm{s})$ were higher than the previous curve, indicating that the shields were performing better than expected and stopping larger projectiles. Both of these effects can be described as a general shift of the SPHC ballistic limit curve toward higher velocities. At least part of the high velocity effect is due to the vaporization of the lead portion of the debris cloud as it hit the back wall and reflected upward to interact with the remainder of the oncoming debris cloud. This is an effect not previously considered. Extending the effects of the vapor phase transition to higher velocities suggests that the ballistic limit curve may remain flat to velocities as high as $50 \mathrm{~km} / \mathrm{s}$.

\section{References}

'Monaghan, J. J., “An Introduction to SPH," Computational Physics Communications, Vol. 48, 1988, p. 89.

${ }^{2}$ Hayashida, K. B. and Robinson, J. H., Single Wall Penetration Equations, NASA TM-103565, December 1991.

${ }^{3}$ Palmieri, D. M., Faraud, M., Destefanis, R., and Marchetti, M., "Whipple Shield Ballistic Limit at Impact Velocities Higher Than $7 \mathrm{Km} / \mathrm{s}$," International Journal of Impact Engineering, Vol. 26, Nos. 1 - 10, December 2001, pp. $579-590$.

${ }^{4}$ Christiansen, E. L., MeteoroidDebris Shielding, NASA TP-2003-210788, August 2003. 